\title{
A DYNAMIC BIOMECHANICAL EVALUATION OF LIFTING MAXIMUM ACCEPTABLE LOADS
}

\author{
Andris Freivalds*, Don B. Chaffint, Arun Garg $\ddagger$ and Kwa. S. LeE† \\ *Department of Industrial and Management Systems Engineering. The Pennsylvania State University. \\ Lniversity Park, PA 16802. U.S.A.: † Center for Ergonomics, University of Michigan. Ann Arbor. \\ MI 4\$109. L.S.A. and $\neq$ Department of Industrial and Systems Engineering. The University of \\ Wisconsin. Milwaukee. WI 53201 . U.S.A.
}

\begin{abstract}
A biomechanical evaluation of the job-related stresses imposed upon a worker is a potential means of reducing the high incidence rates of manual material handling injuries in industry. A biomechanical model consisting of seven rigid links joined at six articulations has been developed for this purpose. Using data from cinematographic analysis of lifting motions the model calculates: (1) body position from articulation angles, (2) angular velocities and accelerations, (3) inertial moments and forces, and (4) reactive moments and forces at each articulation, including the $L_{\mathrm{s}} S_{1}$ joint.

Results indicated effects of the common task variables. Larger load and box sizes increased the rise times and peak values of both vertical ground reaction forces and predicted $L_{5} / S_{1}$ compressive forces. However. boxes with handles resulted in higher $L_{s} / S_{1}$ compressive forces than for boxes without handles. Also, in lifting the larger boxes the subjects did not sufficiently compensate with reduced box weights in order to maintain unitorm $L_{s} S_{1}$ compressive forces. Smoothed and rectitied EMG of erector spinae muscles correlated significantly with $L_{s} / S_{1}$ compressive forces, while predicted and measured vertical ground reaction forces also cerrelated significantly, indicating the validity of the model as a tool for predicting job physical stresses.
\end{abstract}

$\begin{array}{ll}\boldsymbol{\alpha}_{H S} & \text { angle of HS to horizontal (degrees) } \\ \alpha_{S 1 S} & \text { angle of SIS to horizontal (degrees) } \\ \alpha_{L, A} & \text { angle of upper arm to horizontal (degrees) } \\ \alpha_{L L} & \text { angle of upper leg to horizontal (degrees) } \\ \beta & \text { angle between SIS and HS vectors (degrees) } \\ y & \text { angle between HSI and HS vectors (degrees) } \\ \delta & \text { angle between HS1 and S1S vectors (degress) }\end{array}$

\section{INTRODLCTION}

Automation has significantly reduced the demands for human strength in the modern industrial environment. Nonetheless, muscular power still remains an essential part of many occupations, particularly those involving manual materials handling ( $\mathrm{MMH}$ ) or maintenance work. In these activities, the moving of heavy loads can cause high stresses on the musculo-skeletal system resulting in $25 \%$ of all lost time in industrial accidents (National Safety Council, 1979).

A large part of this cost is due to low back incidents since this injury often results in a permanent disorder causing considerable discomfort and limitations for the employee and a large expense for the employer. In Great Britain, as high a $20^{\circ}$ of reported accidents in industry are back injuries (Troup, 1965) and of which $5060^{\circ}$ result from lifting. As is usually the case, an accurate description of the lifting motion causing the injuries is not known. If the motion is fast and "jerky", the inertial forces on the body are increased due to acceleration. If the back is over-flexed, the ligaments of the spine can be strained. If rapid acceleration of the torso occurs, stresses on the spine are compounded. Furthermore, the task variables, such as the size, the shape and the weight of the load, influence the stress on 
the back. Consequently, there is a need to specify and quantify the characteristics of lifting tasks in an attempt to reduce this serious occupational health problem.

Such quantification has been attempted previously with dynamic biomechanical modeling. Much of the early work was performed by Pearson et al. (1963), who performed a dynamic analysis of the human upper extremity. Plagenhoef (1966) pioneered the methods of obtaining and modeling kinematic data for wholebody human motion. The inclusion of the calculations of spinal compressive forces (Fisher, 1967) with a dynamic analysis of lifting motion patterns in the sagittal plane (Chaffin, 1969) formed the bases for the model presented in this paper. After an initial analysis of MMH activities (Chaffin and Baker, 1970), the model was separated into two versions: a threedimensional, static strength prediction model discussed in Garg and Chaffin (1975), Chaffin et al. (1977), and Freivalds (1980), and a dynamic sagittal plane kinematic version to be discussed here. An alternative approach to dynamic biomechanical modeling has also been developed by Ayoub and El-Bassoussi (1978), and involves predicted movement dynamics based on acceleration patterns modeled by Slote and Stone (1963).

The general purpose of this report is to describe the development of a methodology for analyzing the amount of stress imposed on a person's musculoskeletal system, especially the low back, during infrequent MMH tasks. More specifically there are three objectives: (1) to detail the implementation of a dynamic biomechanical model using actual segment motion data, (2) to investigate the effect of box size on the weight of the load selected, i.e., whether the worker will compensate for larger box size with lighter loads in order to maintain uniform low back compressive loads, and (3) to validate the model by comparing predicted and measured ground reaction forces and by correlating predicted low-back compressive forces external, semi-direct indicators of load, obtained from surface electromyography of the erector spinae muscles measured during the lifts. Once adequately validated, the model can be used to evaluate physically limiting situations in industry and to propose alternative courses of action.

\section{MATERIALS AND METHODS}

\section{The biomechanical model}

The basic assumption behind the dynamic biomechanical model is that the body is made up of rigid links joined at known, simple articulations. This is certainly valid for the arms and legs, but less so for the trunk which is a semiflexible arrangement of vertebral bodies, intervertebral discs and cartilaginous endplates located between the vertebral bodies and discs. Seven links are used: (1) hand-forearm, (2) upper arm, (3) thoracic-lumbar, (4) pelvis, (5) upper leg. (6) lower leg and (7) foot. The trunk division into two links is at the $L_{s} / S_{1}$ level to allow the calculation of spinal compressive forces and moments at this disc which is most often injured during lifting. The ankle is assumed to remain in a fixed planar position, which provides a constant reference. Finally, the wrist is not treated as an articulation as there is very little wrist-action involved in gross planar motions. A schematic representation of the model is shown in Fig. 1.

There are four steps in resolving moments and forces on the body from the motion input data (i.e. the $X, Y$ joint position data over time for the ankle, knee, hip, shoulder, elbow and hand $c g$ ). The steps are: (1) the resolution of the position of the body from the angles at each articulation; (2) the determination of the angular velocities and angular accelerations at each articulation, which in turn, gives the linear acceleration of the body links; (3) the calculation of inertial forces and inertial resistance moments due to acceleration; and (4) the calculation of reactive moments and forces at each articulation exerted by the muscles to overcome the resultant forces due to external loads and body weight. These steps are explained in more detail in the Appendix.

\section{Operating the biomechanical model}

There are three types of input to the biomechanical model: (1) the mass and length of the links of the body, and (2) a description of the motion of each link for the lifting action, and ( 3 ) the load being moved. The weight of each body link has been correlated with gross body weight. Average values have been tabulated by Drillis and Contini (1966) and were used in the model as a proportion of the subject's body weight. The location of $\mathrm{cgs}$ as function of link lengths were used from the data of Dempster (1955). The lifting motion was put in as a series of joint coordinates over time for the ankle, knee, hip, shoulder, elbow and hand $\mathrm{c} g$ articulations.

\section{Subjects}

Six male subjects participated in the study. All were paid volunteers and students in good health. All had been informed of the experimental protocol and risks and had given written consent prior to participation. Their ages, body weight, stature and isometric torso lifting strengths, with corresponding percentiles are given in Table 1. Height and weight percentiles are calculated from U.S. Department of HEW (1979) and strength percentiles from Chaffin et al. (1977).

\section{Equipment}

The subjects stood on a six-axis Kistler force platform which was connected on-line to an HP-2100 minicomputer. Monopolar surface electromyograph (EMG) recordings were obtained from the erector spinae muscles at the $I_{3} / I_{4}$ level. Beckman silver/silverchloride electrodes were applied to the abraded areas and allowed to set in until the DC resistance was below $50 \mathrm{k} \Omega$. The EMG signals were first amplified by a differential preamplifier with approximately infinite input impedence and were then conditioned by a Heath-Schlumberger $\mathrm{AC}$ voltmeter which acted as a 


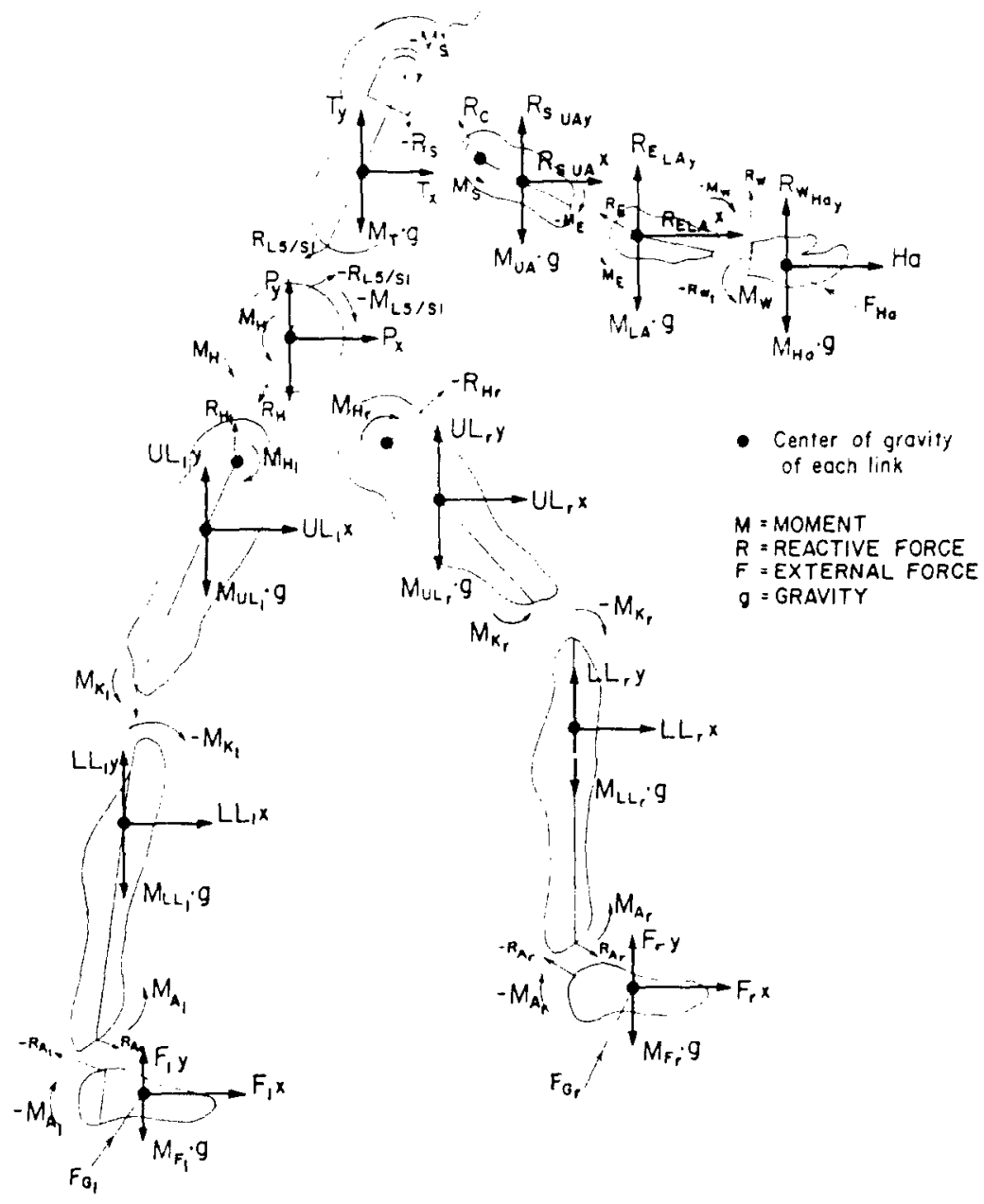

Fig. 1. Free body diagram of the human body.

rectifier and smoother of the EMG signal. The conditioned signal was digitized at $40 \mathrm{~ms}$ intervals and entered on-line to the minicomputer.

A $35 \mathrm{~mm}$ camera and a stroboscopic light flashing at
$20 \mathrm{~Hz}$ were set at about $3 \mathrm{~m}$ from the subject as shown in Fig. 2. The subject wore black leotards and reflective markers were attached over each of the major joints. The camera shutter was opened and kept open for the

Table 1. Subject attributes

\begin{tabular}{|c|c|c|c|c|c|c|c|}
\hline \multirow[t]{2}{*}{ Subjict } & \multirow{2}{*}{$\begin{array}{l}\text { Age } \\
(y r)\end{array}$} & \multirow{2}{*}{$\begin{array}{l}\text { Body } \\
(\mathrm{kg})\end{array}$} & \multirow{2}{*}{$\begin{array}{l}\text { mass } \\
(", \text { tile) }\end{array}$} & \multicolumn{2}{|c|}{ Stature } & \multicolumn{2}{|c|}{$\begin{array}{l}\text { Isometric torso } \\
\text { lifting strengths }\end{array}$} \\
\hline & & & & $(m)$ & (" ", tile) & $(N)$ & (" "tile) \\
\hline$J C$ & 23 & 74 & 54 & 1.71 & 30 & 347 & 59 \\
\hline$D F$ & 21 & 85 & 88 & 1.79 & 70 & 520 & 91 \\
\hline$M P$ & 23 & 80 & 76 & 1.83 & 72 & 249 & 24 \\
\hline$J R$ & 18 & 62 & 12 & 1.75 & +9 & 200 & 9 \\
\hline$B S$ & 29 & 73 & 46 & 1.85 & 94 & 298 & 43 \\
\hline$D Z$ & 24 & 60 & 8 & 1.75 & $\$ 9$ & 267 & 31 \\
\hline
\end{tabular}




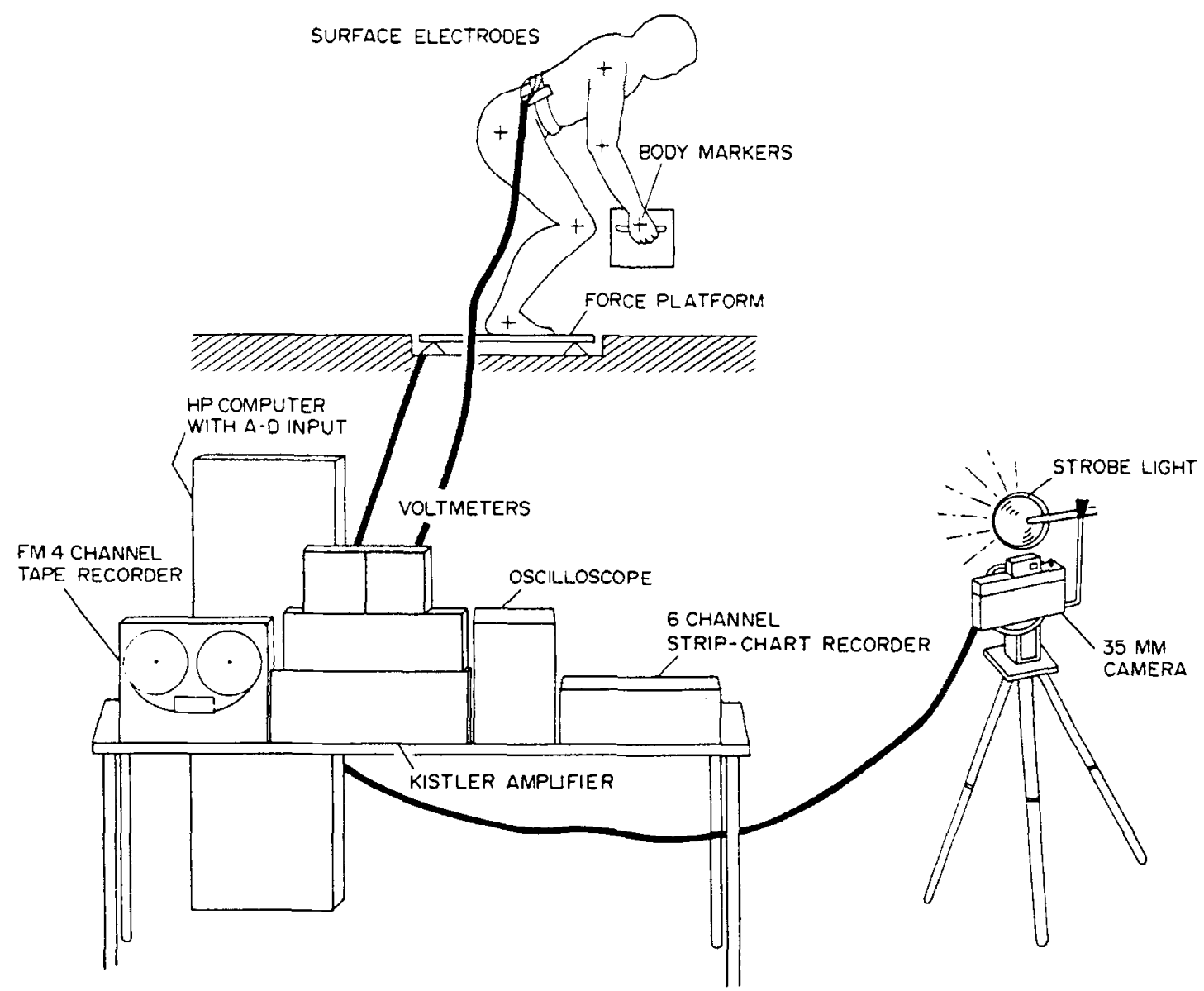

Fig. 2. Schematic drawing of experimental set-up.

duration of the motion while the strobe lights were activated by a micro-switch as soon as the motion started.

\section{Procedure}

Prior to testing, each subject demonstrated maximum isometric torso, leg and arm strengths according to the specifications of Chaffin et al. (1977). Each subject was then asked to lift six repetitions of four different boxes, holding various weights. The boxes used were $0.46 \mathrm{~m}$ wide but varied in length (i.e. extending forward from the toes). The four combinations were as follows:

$$
\begin{aligned}
& 0.46 \mathrm{~m} \times 0.38 \mathrm{~m} \text {-with handles } \\
& 0.46 \mathrm{~m} \times 0.51 \mathrm{~m} \text {-with handles } \\
& 0.46 \mathrm{~m} \times 0.64 \mathrm{~m} \text {-with handles } \\
& 0.46 \mathrm{~m} \times 0.38 \mathrm{~m} \text {-without handles. }
\end{aligned}
$$

The loads carried in the boxes were selected psychophysically (per Snook. 1978). Essentially, each subject was allowed to select the heaviest weight he would lift determined by his own feelings of exertion or fatigue. He was asked to do this for every box type, thereafter performing three lifts with that load. He then selected another psychophysical load for the same box and performed three additional lifts. He then progressed to another type of box repeating the procedure. For each lift, the subject started with the box on the floor directly in front of the toes and lifted the box in a true posture to a table at waist height. In all cases, the subject was given adequate rest between lifts. The total experimental session lasted about two hours per day for four days.

\section{ANALYSIS}

The raw data from the force platform and EMG amplifiers were digitized at equal intervals and stored on magnetic tape for further analysis. The lifting motion was decoded from the $35 \mathrm{~mm}$ slide containing the stroboscopic movement. The joint locations were digitized from an enlargement of the slide using a Graf-Pen Sonic digitizer connected to a HewlettPackard 9830 desk-top computer.

An example of the digitized data is presented in Fig. 3 with the locations for ankle, knee, hip, shoulder, elbow and hand $c g$ indicated. For clarity, articulations at equal time intervals are connected by straight lines to give the stick figure effect. Successive points are 


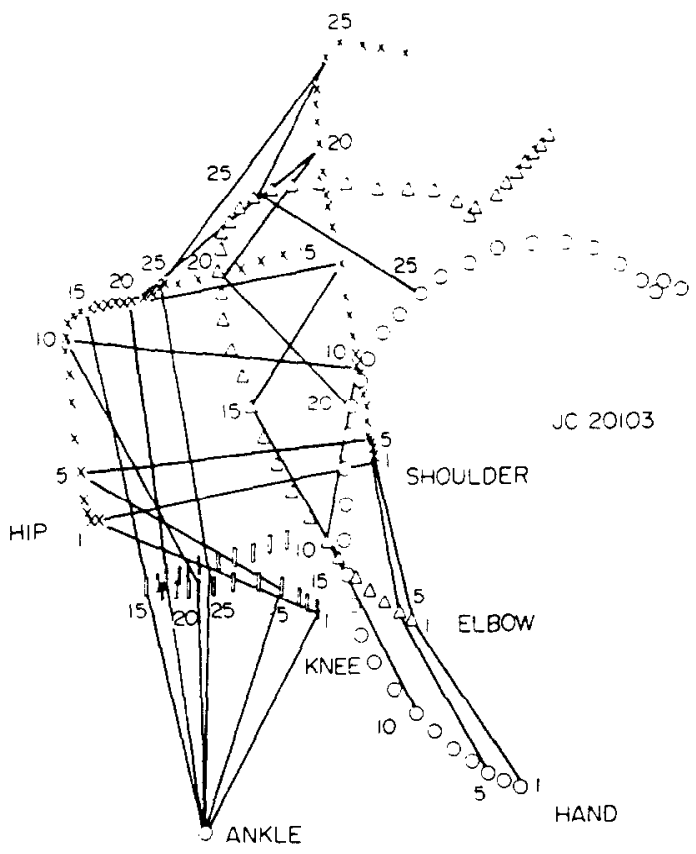

Fig. 3. Stroboscopic motion.

separated by $50 \mathrm{~ms}$ intervals. These data were then input to the dynamic biomechanical model.

The output of the model included the following for each time period:

(1) elapsed time,

(2) angle of each link,

(3) angular velocity about each articulation,
(4) accelerations in $X$ and $Y$ directions of the $c g$ for each link.

(5) reactive forces in $X$ and $Y$ directions at each articulation,

16) reactive torques at each articulation.

Besides the above information. the compressive and shear forces at the $L_{5} / S_{1}$ articulation were also output. Finally, plots of acceleration, velocity and displacement in $X$ and $Y$ directions for all articulations were presented.

The digitized vertical ground reaction $(Y)$ forces from the force platform and rectified EMG along with the predicted vertical ground reaction forces and predicted compressive forces at the $L_{5} S_{1}$ articulation were further analyzed statistically. Plots, correlations, analysis of variance and various other statistical measures were performed.

\section{RESULTS}

The results of the analysis will be presented in the following arrangement: (1) results of the vertical ground reaction $(Y)$ forces including various subject and task variable effects, (2) results of the predicted vertical ground reaction forces including correlations with the measured ground reaction forces, (3) results of predicted compressive forces including correlations with the vertical ground reaction forces and (4) results of rectified EMG including correlations with back compressive forces.

A plot of the vertical ground reaction forces, as measured from the $Y$ force of the force platform vs time into lift, is shown individualy and averaged over all subjects and all conditions in Fg. 4. The initial value

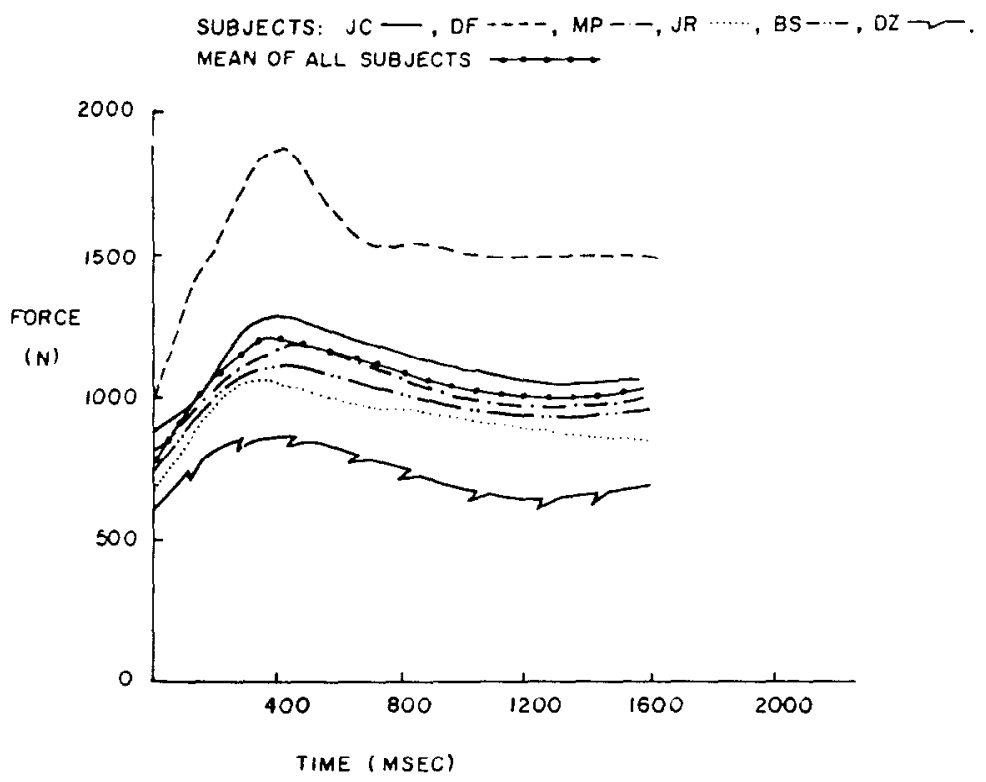

Fig. 4. Vertical ground reaction forces--individual subjects. 
Table 2. Maximum volitional loads chosen

\begin{tabular}{ccccccccc}
\hline & \multicolumn{2}{c}{$38 \mathrm{~cm}$} & \multicolumn{2}{c}{$51 \mathrm{~cm}$} & \multicolumn{2}{c}{$64 \mathrm{~cm}$} & \multicolumn{2}{c}{$38 \mathrm{~cm}$ no handles } \\
Subject & Test 1 & Test 2 & Test 1 & Test 2 & Test 1 & Test 2 & Test 1 & Test 2 \\
\hline$J C$ & 431 & 416 & 342 & 347 & 311 & 320 & 387 & 420 \\
$D F$ & 567 & 533 & 496 & 498 & 547 & 536 & 565 & 662 \\
$M P$ & 216 & 260 & 222 & 227 & 191 & 213 & 282 & 258 \\
$J R$ & 365 & 342 & 240 & 329 & 222 & 200 & 307 & 302 \\
$B S$ & 218 & 220 & 224 & 222 & 211 & 222 & 269 & 269 \\
$D Z$ & 178 & 149 & 149 & 160 & 131 & 138 & 102 & 133 \\
\hline
\end{tabular}

at $t=0$ corresponds to the subject's weight. The rise in the vertical ground reaction forces corresponds to the effect of accelerating the load and peaks at about $400 \mathrm{~ms}$ at values as much as $40 \%$ greater than the static load. Thereafter, the forces decrease and level off at a value that is equivalent to the subject's weight plus the load.

The variations in the pattern between subjects are mainly due to peak values which depend on the subject's mass, load selected (see Table 2 ) and acceleration of the load during the lift. In fact, this force varies between $1864 \mathrm{~N}$ for subject $D F$ to $868 \mathrm{~N}$ for subject $D Z$, a more than two-fold difference. This difference can be mostly acounted for by the $245 \mathrm{~N}$ of added body weight of subject $D F$ and by the $409 \mathrm{~N}$ of added average load subject $D F$ chose. The time to peak varies also between subjects $(350-420 \mathrm{~ms})$ but is not nearly as dramatic.
A plot of the measured vertical ground reaction forces (averaged for all subjects) vs time into lift as a function of the type of container is shown in Fig. 5. The patterns for the $0.51 \mathrm{~m}$ boxes and $0.64 \mathrm{~m}$ boxes with handles are very similar for the $0.38 \mathrm{~m}$ box with handles and are removed for visual clarity. The graphs show the paradoxical situation of a faster rise time and higher peak force for the box with handles than for the box without handles even though the larger loads were handled for boxes without handles.

A plot of predicted ground reaction forces vs time into lift is shown in Fig. 6 superimposed on the graph for measured ground reaction forces. The predicted forces peak at $1241 \mathrm{~N}$ compared to $1234 \mathrm{~N}$ for the measured forces. However, the rise time for the predicted is slightly faster, at a little more than $300 \mathrm{~ms}$ to about $390 \mathrm{~ms}$ for the measured. The average
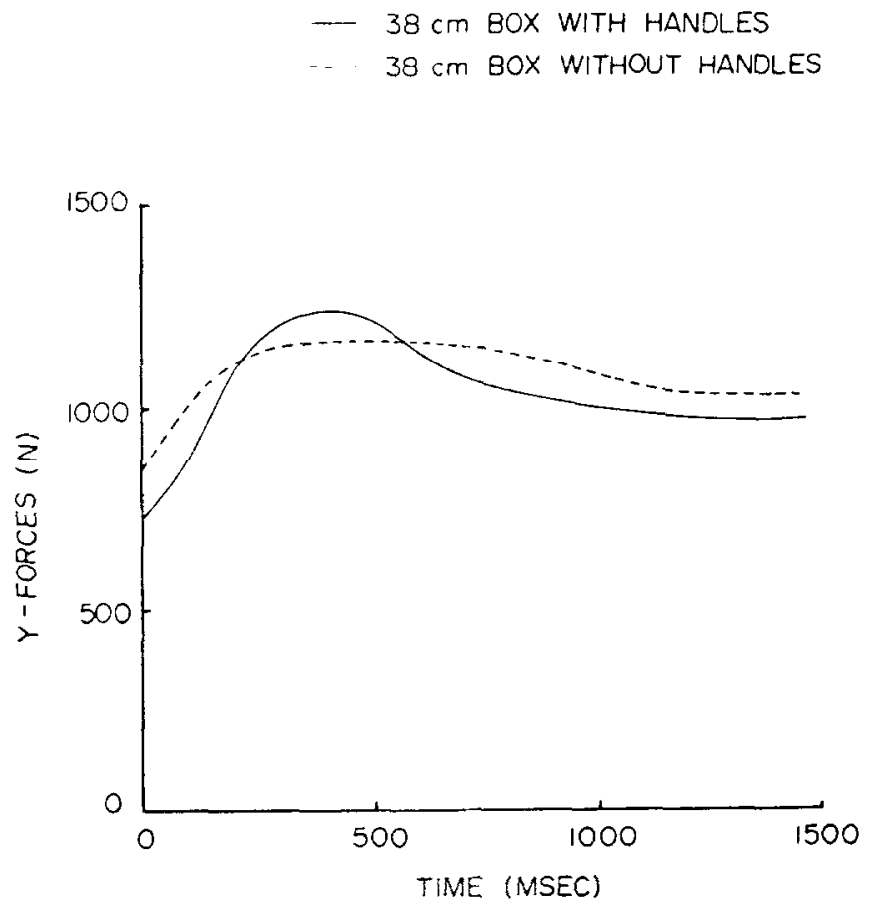

Fig. 5. Vertical ground reaction forces $-38 \mathrm{~cm}$ boxes with and without handles. 


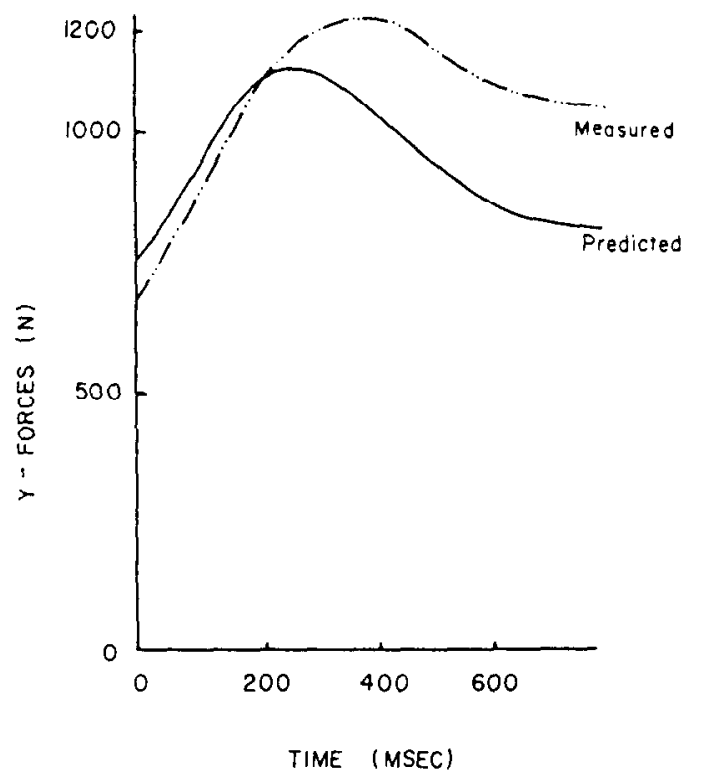

Fig. 6. Predicted and measured ground reaction forces $(n)$.

correlation between predicted and measured ground reaction forces over time for all subjects and boxes is 0.43 , which is significant at $p<0.05$. Lower than expected correlations could have resulted from errors in the synchronization of the strobe camera with the on-line digitizing of the force and EMG data. Initial movements of the subjects such as shifting the box could have mistriggered the synchronized sequence.

A plot of predicted $L_{5} / S_{1}$ compressive forces vs time into lift is shown in Fig. 7. This series of curves is averaged over subjects to show box effects. These patterns are somewhat different from the vertical ground reaction forces in that all of the back compressive force curves tend to have faster rise times indicating greater link and load accelerations from rotational effects. Also, there tend to be slight dips, even double peaks for the larger boxes with handles. For the $0.64 \mathrm{~m}$ box, this appears almost to be oscillatory.

Furthermore, larger box sizes do not necessarily imply larger compressive forces. In fact, the $0.51 \mathrm{~m}$ and $0.64 \mathrm{~m}$ boxes showed smaller peak forces than the $0.38 \mathrm{~m}$ box. This could be accounted for by the decreased maximum loads selected by the subjects for the larger boxes. The average load selected for the $0.38 \mathrm{~m}$ box was $321 \mathrm{~N}, 0.51 \mathrm{~m}$ box $-289 \mathrm{~N}$ and $0.64 \mathrm{~m}$ box-271. However, the $0.38 \mathrm{~m}$ box without handles showed lowest compressive forces, even though the average load was greatest, at $334 \mathrm{~N}$.

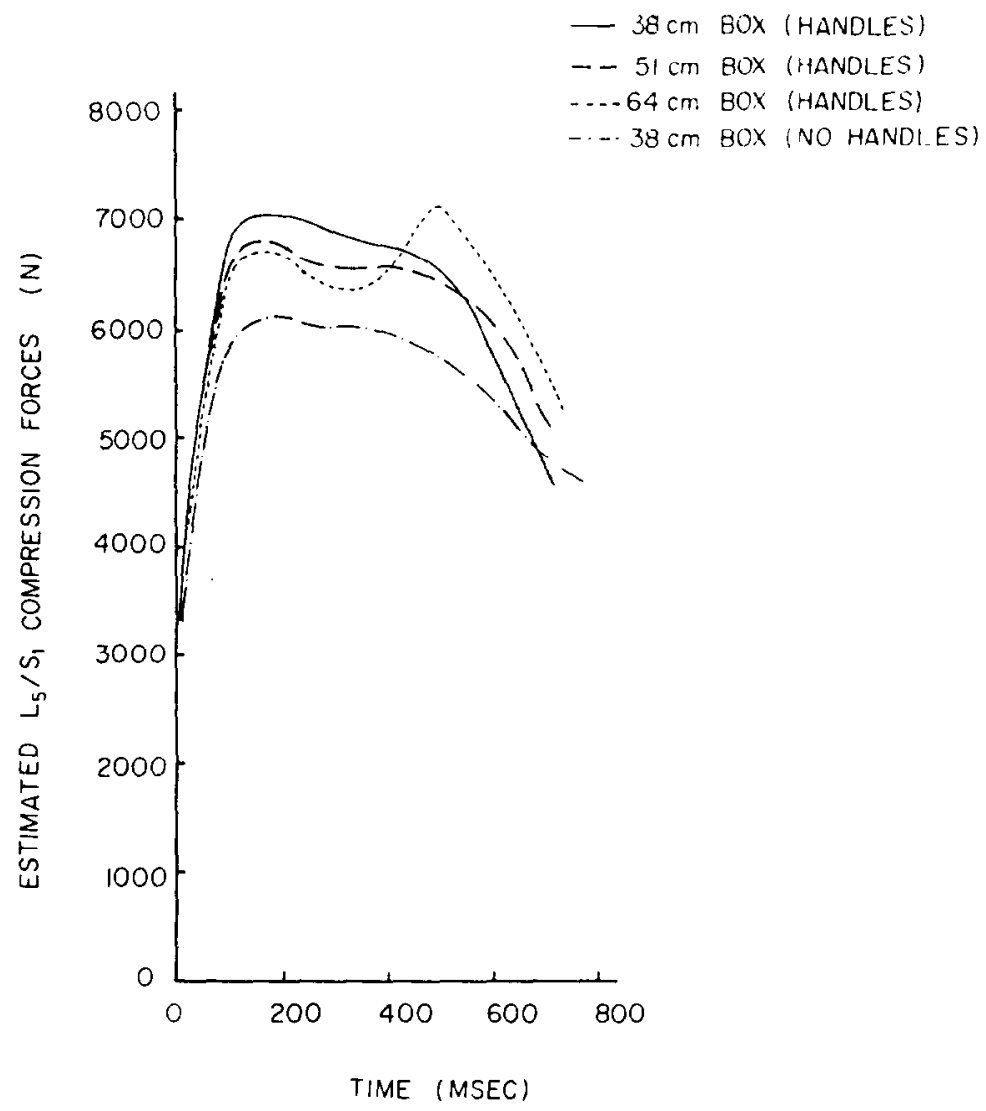

Fig. 7. Predicted $L_{s} / S_{1}$ compressive forces. 
A more perceptive analysis is to normalize the peak $L_{5} / S_{1}$ compressive force for each trial with the load handled, $F_{\text {comp }}$ load. A two-way analysis of variance of these values yielded significant subject effects $(p<0.001)$ and significant box effects $(p<0.01)$. The subject effects are due to widely different isometric torso strengths (Table 1).

The box effects are more interesting. Larger boxes had significantly larger ratios, as would be expected from the increased moment arm for a given amount of load. However, the $0.38 \mathrm{~m}$ box without handles had a smaller $F_{\text {comp }} /$ load ratio than the $0.38 \mathrm{~m}$ box with handles.

Plots of predicted $L_{5} / S_{1}$ compressive forces averaged across boxes to show individual effects were similar to the vertical ground reaction forces of Fig. 4 . However, the compressive forces showed faster rise times. As expected, the subjects handling heaviest loads exhibited also the largest compressive forces. In fact, the average correlation coefficient between peak compressive force and the load handled for all trials is 0.7 which is significant at $p<0.001$.

A plot of the smoothed and rectified EMG of the erector spinae muscles vs time into lift is shown in Fig. 8. This is an average of all subjects and all boxes. The individual curves follow a similar shape with slightly different peak values. In general, the EMG starts from a tonic level and increases at about the same rate as the low back compressive forces. Correlation of compressive forces and the EMG over time for all trials yields an average correlation coefficient of $r=0.42$, significant at $p<0.05$.

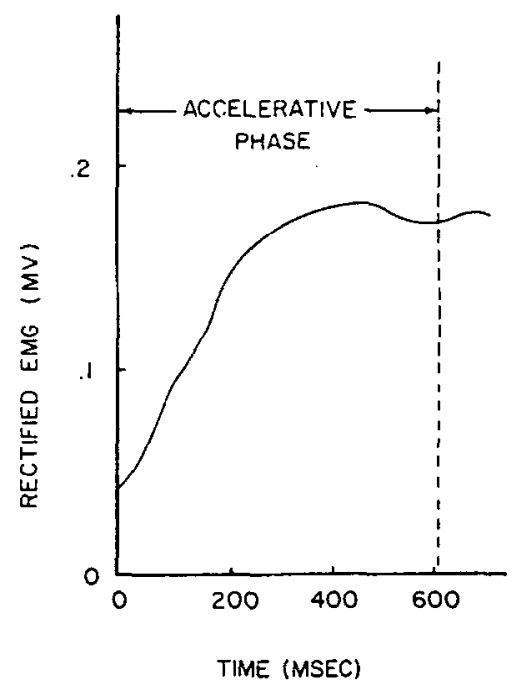

Fig. 8. Smoothed and rectified EMG.

\section{Discl'ssion}

The results confirmed several logical principles concerning the effect of task variables on lifting dynamics. Larger loads act to increase vertical ground reaction forces and, more importantly, $L_{5} / S_{1}$ compressive forces. Average correlations for loads with corresponding peak vertical ground reaction forces $(r=0.91)$ and for loads with corresponding peak compressive forces $(r=0.7)$ are both significant. The dynamic effects, overcoming with initial moments of inertia, acceleration patterns, deceleration patterns, etc. also tend to increase vertical ground reaction forces and the $L_{s} / S_{1}$ compressive forces. This effect amounts to increasing the static load by as much as $40 \%$ of its weight. Undeniably, the faster or jerkier the lifting motion, the greater the compressive forces and the greater the chance for injury.

Larger box sizes create larger moment arms and increase $L_{s} / S_{1}$ compressive forces as would be expected and as confirmed by the two-way analysis of variance of $F_{\text {comp }} /$ load ratios. Unfortunately these increases tend to indicate that a normal person is unable to compensate for an increased box size by an appropriate reduction in the load to maintain uniform compressive forces. In fact, relying on a worker's perception of the stresses of lifting a load may not be adequate to protect the person. On the other hand, a possible internal safeguard is shown by a closer examination of the compressive forces over time for the various sized boxes which reveals oscillatory lifting motions. A possible explanation could be the parabolic lifting trajectory used by most subjects. Upon grasping the box, the subjects had a tendency of leaning back and pulling the box into the waist, so as to maintain the whole body plus load (e.g. over the feet as much as possible). At the end of the lift while setting the box on the shelf, the subjects would then push the box away from the body. In effect, this process causes the moment arm of the load acting on the $L_{5} / S_{1}$ joint to decrease from an initial value and then to increase as the box is set on the shelf, thus creating the oscillatory pattern in the back compressive force.

It could be assumed that handles would reduce the chance for slips and jerky motions, reduce $F_{\text {comp }}$ and thus create a safer means of handling the load. However, the opposite effect was observed. Both vertical ground reaction forces and $L_{5} / S_{1}$ compressive forces showed faster rise times and larger peaks for the $0.38 \mathrm{~m}$ box with handles than without handles even though larger loads were handled than for boxes without handles $(329 \mathrm{~N}$ vs $324 \mathrm{~N})$. Similarly the $F_{\text {comp }} /$ load ratios showed significantly lower values for the boxes without handles. This paradox suggests that all handles on containers might be eliminated in order to produce lower stress on the musculoskeletal system during a lifting motion, although being more hazardous in that the box may slip from one's grasp. In short, it appeared that the subjects realized the hazard and were more cautious in their lifting motion. 
Similar factors have been examined empirically in the studies of Konz and Desai (1976) and Coetzee and Konz (1978) using ground force reactions from a force platform only. Even though different experiment protocols were followed from the present study, generally similar results were found. Konz and his group used much lower weights resulting in much smaller vertical ground reaction forces than in the present study. Furthermore. Konz and his subjects used more elaborate lifting procedures resulting in longer and more oscillatory lifting patterns corresponding to the down-up movements of the subjects. However, they still found peak vertical ground reaction force occurring about $\mathbf{4 0 0} \mathrm{ms}$ after lift-off. and vertical ground reaction forces higher for heavier loads and larger ground reaction moments for larger boxes. The last compares favorably with increased back compressive forces for the larger box sizes of the present study.

The results also confirmed the predictive capabilities of a dynamic biomechanical model. Predicted and measured vertical ground reaction forces correlated significantly, exhibited the same peaks and differed only slightly in rise times. Predicted $L_{s}, S_{1}$ compressive forces correlated significantly with vertical ground reaction forces, thus not only aiding in the validation of the model but also providing a means of estimating back compressive forces.

Additional validation was obtained by comparison of predicted low-back compressive forces with EMG of the erector spinae muscles, a method also used by Andersson et al. (1980) for static exertions. Smoothed and rectified EMG correlated significantly $(p<0.05)$ with $L_{5} / S_{1}$ compressive forces confirming the use of spinal mechanics in the model. The findings also imply that in similar situations, myoelectric activity can be used as a direct indication of muscle contraction force and low-back compressive forces during dynamic lifting.

Thus, a working biomechanical model has been developed and used to determine the relative stresses of various types of lifting motions. The use of such biomechanical analysis in industry is a needed tool to provide a means of reducing the job related stresses imposed upon the worker and eliminating some of the high costs of hazardous manual materials handling activities.

\section{CONCLLSIONS}

(1) Vertical ground reaction forces and predicted $L_{5} / S_{1}$ compressive forces increased with increasing load.

(2) Vertical ground reaction forces and predicted $L_{s} / S_{1}$ compressive forces increased with increasingly larger boxes.

(3) Vertical ground reaction forces and predicted $L_{5} / S_{1}$ compressive forces exhibited slower rise times and lower peak forces for boxes without handles, indicating more controlled motion.
(4) Smoothed and recitified EMG correlated significantly with predicted $L_{5} S_{1}$ compressive forces.

(5) $L_{3} S_{1}$ compressive forces correlated significantly with loads handled.

(6) Predicted vertical ground reaction forces correlated significantly with measured vertical ground reaction forces, indicating the validity of the model.

\section{REFERENCES}

Andersson. G. B. J.. Ortengren, R. and Schultz, A. ( 980 ) Analysis and measurement of the loads on the lumbar spine during work at a table. J. Biomechanics 13, 513-520.

Ayoub, M. M., Bethea. M. J., Deivanayagam. S., Asfour, S. S., Bakken, G. M., Liles, D., Mital, A. and Sherif, M. (1978) Determination and modeling of lifting capacity. Technical Report. Texas Technical University.

Ayoub. M. S. and El-Bassoussi. M. S. (1978) Dynamic biomechanical model for sagittal plane lifting activities. Safety in Manual Materials Handling (Edited by Drury, C. G.) $78-185 \mathrm{NIOSH}$, Cincinnati, $\mathrm{OH}$

Bartelink, D. L. (1957) The role of abdominal pressure in relieving the pressure on the lumbar intervertebral discs. J. Bone Jt Surg. 39B, 718.

Chaffin, D. B. (1969) A computerized biomechanical modeldevelopment and use in studying gross body actions J. Biomer hanics 2, 429-441

Chaffin, D. B. and Ayoub, M. M. (1976) The problem of manual materials handling. Professional Safety 21, 28-30.

Chaftin. D. B. and Baker, W. H. (1970) A biomechanical model for analysis ov symmetric sagittal plane lifting. $A I I E$ Trans., 2, 16-27.

Chaffin, D. B., Fisher, B., Hodges, W. and Miyamato, R. (1967) A biomechanical analysis of materials handling activities performed in the sagittal body plane. Research Report. The University of Michigan.

Chaffin, D. B., Herrin, G. D., Keyserling, W. M. and Foulke, J. A. (1977), Pre-Employment Strength Testing in Selecting Workers for Materials Handling Jobs, DHEW No. 77-163. NIOSH, Cincinnati, $\mathrm{OH}$.

Chaffin, D. B., Herrin, G. D., Keyserling, W. M. and Garg, A. (1977) A method for evaluating the biomechanical stresses resulting from manual materials handling jobs. Am. ind. Hyg. Ass. J. 38, 662-675.

Coetzee, J. and Konz, S. (1978) Force platform output for manual lifting as a function of task, technique and individual variables. Proceedings of the Human Factors Society, 22nd Annual Meeting. Human Factors Society.

Davis, P. R. and Troup, J. D. G. (1964) Pressures in the trunk cavity when pulling, pushing and lifting. Eryonomics 7 . 465-474.

Dempster, W. T. (1955) Space requirements of the seated operatur, WADC Technical Report 55-159. University of Michigan.

Dempster, W. T., Sherr, L. A. and Priest, J. G. (1964) Conversion scales for estimating humeral and femoral lengths and the lengths of functional segments in the limbs of American caucasoid males. Hum. Biol. 36, 246-261.

Drillis. R. and Contini. R. (1966) Body segment parameters. Technical Report No. 1166.03, School of Engineering and Science, New York University.

Fisher, B. O., Jr. (1967) Analysis of spinal stress during lifting, a biomechanical model, unpublished M.S. Thesis, The University of Michigan.

Freivalds, A. (1980) The development and use of biomechanical strength models. NATO Symposium on Anthropometry and Biomechanics, 7-11 July, 1980. Cambridge, U.K. Plenum Press, New York.

Garg, A. and Chaffin, D. B. (1975) A biomechanical computerized simulation of human strength. AIIE Trans. 7 , $1-10$. 
Halze. H. (1981) The use of optimally regularized fourier series for estimating higher order derivatives of noisy biomechanical data. J. Biomechanics 14, 13-18.

Konz, S. and Desai, C. (1976) Lifting forces for nine lifting heights. Agressologie 17, 55-59.

Lanier. R. R. (1939) Presacral vertebrae of white and negro males. Am. J. Phys. Anthropol. 25, 343-20.

Martin. J. B. and Chaftin. D. B. (1972) Biomechanical computerized simulation of human strength in sagittal plane activities. AIIE Trans. 4, 19-28.

Morris. J. M.. Lucas. D. B. and Bressler, B. (1961) Role of the trunk in stability of the spine. $J$. Bone $J_{t}$ Surg. 43 A. $327-351$

National Safety Council (1979) Acciden Facts. Chicago.

Pearson, J. R., McGinley, D. R. and Butzel. L. M. (1963) Dynamic analysis of the upper extremity for planar motions. Hum. Factors 5, 59-70.

Perey, O. (1957) Fracture of the vertebral end plate in the Lumbar spine, an experimental biomechanical investigation. Acta orthop. scand: Suppi. 25.

Plagenhoef, S. C. (1966) Methods for obtaining kinematic data to analyze human motions. Res. Q. 37, 103-112.

Schanne, F. J.. Jr. (1972). A three-dimensional hand force capability model for a seated operator. Ph.D. Thesis. The University of Michigan.

Slote, L. and Stone, G. (1963) Biomechanical power generated by forearm flexion. Hum. Factors 5, 443-452.

Snook. S. H. (1978) The design of manual handling tasks. Ergonomics 21, 963-985.

Troup, J. D. G. (1965) Relation of Lumbar Spine Disorders to Heavy Manual Work and Lifting. Lancet, April 17, 857-861.

U.S. Department of HEW (1979) Weight and height of adults, 18-14 years of age, 1971-1974. DHEW Publication No. 79-1659, National Center for Health Statistics, Hyattsville. MD.

Winter, D. A., Sidwall, H. G. and Hobson. D. A. (1974) Measurement and reduction of noise in kinematies of locomotion. J. Biomechanics 7. 157-159.

Zernicke, R.F., Caldwell, G. and Roberts, E. M. (1976) Fitting biomechanical data with cubic spline functions. Res. Q. 47. 9-19.

\section{APPENDIX. THE BIOMECHANICAL MODEL}

The first computation involves the resolution of the body position from the angles at each articulation by creating a series of vectors corresponding to the links between the articulations. One complexity arises in inserting the $L_{5} / S_{1}$ joint between the hip and shoulder joint. Due to curvature of the spine, the $L_{5} / S_{1}$ disc can fall posterior to the line connecting the shoulder and the hips. To add this articulation involves several assumptions. First, in the vertical erect posture, the $L_{5} / S_{1}$ joint is assumed to be $19.5 \%$ above thu distance between the hip and shoulder (Lanier, 1939). Second, the amount of forward pelvic rotation relative to lumbar rotation is determined by the amount of trunk flexion. A study by Dempster et al. (1964), indicated that for the first $27^{\circ}$ of trunk rotation, the pelvis does not significantly rotate, i.e. most of the rotation occurs in the lumbar spine. Thereafter. the pelvis rotates $2 / 3^{3}$ for every degree of trunk rotation with the remaining $1 / 3$ accounted for by spinal rotation. Thus the pelvic rotation angle pelrot in Fig. 9 is

$$
\text { petrot }=2 / 3\left(x_{\ell L}-x_{H S}-27^{\circ}\right)
$$

where $x_{L L}$ is the thigh angle with respect to horizontal and $x_{H S}$ is the hip-to-shoulder vector to horizontal. Therefore. the pelvic angle is

$$
\text { peltic }=x_{\ell L}-\text { pelrol }
$$

Further computational equations can be found in Chaffin (1969).
For the second step. the angular velocities and angular accelerations can be determined by numerical analysis. Considering any angle of the body at the $j$ th time period. $x$, if that angle and all its time $\dot{x}_{j}$ derivatives are known. $\mathrm{d} x_{j} \mathrm{~d} t$ $=\dot{x}_{j}$ (angular velocity). $\mathrm{d}^{2} \boldsymbol{x}_{j}, \mathrm{~d} t^{2}=\ddot{x}$. (angular acceleration. etc.). the Taylor series gives the angle at the preceding and succeeding time periods

$$
\begin{aligned}
& x_{i+1}=x_{j}+\Delta T x_{j}+\left(\frac{\Delta T}{2}\right)^{2} \ddot{x}_{j}+\ldots \\
& x_{j-1}=x_{j}-\Delta T \dot{x}_{j}+\left(\frac{\Delta T}{2}\right)^{2} \ddot{x}_{j}-\ldots
\end{aligned}
$$

With $\Delta T$ finite and normal situations. an estimate for $\dot{x}_{j}$ and $\ddot{x}_{j}$ can be found by subtracting the above equations and solving for $\dot{x}_{j}$ and $\ddot{x}_{j}$

$$
\begin{aligned}
& \dot{x}_{j}=\frac{x_{j+1}-x_{j-1}}{2 \Delta T} \\
& \ddot{x}_{j}=\frac{x_{j+1}+x_{j-1}-2 x_{j}}{(\Delta T)^{2}} .
\end{aligned}
$$

However, the accelerations tend to be 'jerky'. probably as a result of double differentiating any noise superimposed on the joint data. To alleviate the problem. a smoothing technique using a wider span of time periods was used in the calculation of $\dot{\alpha}_{j}$ and $\ddot{\alpha}_{j}$

$$
\begin{aligned}
& \dot{x}_{j}=\frac{\alpha_{j+2}-x_{j-2}}{4 \Delta T} \\
& \ddot{x}_{j}=\frac{\alpha_{j+3}-x_{j-3}-2 x_{j}}{(3 \Delta T)^{2}} .
\end{aligned}
$$

Other techniques such as cubic spline functions (Zernicke et al., 1976), digital filtering (Winter et ul.. 1974) or Fourier series expansion (Hatze, 1981) may be better techniques and are being incorporated into further model updates.

For the third step, the angular motion kinematic formulae give linear accelerations of any point (at distance $r$ ) on the rotating link

$$
\begin{aligned}
a_{1} & =\ddot{\alpha}_{r} r \text { tangential acceleration } \\
a_{r} & =\dot{\alpha}_{r}^{2} r \text { normal acceleration } \\
a & =a_{t}+a_{r} \text { linear acceleration. }
\end{aligned}
$$

Calculation of accelerations at both the articulation and link $\operatorname{cgs}$ is needed. Relative to the axis of rotation, this can be found by equations (9-11). However, if the axis is also accelerating, the acceleration of the axis must be added to $a$, the linear acceleration, to find the acceleration of the point in the whole reference frame. For example, if the hip linear acceleration relative to the knee is $a_{n}$ based on the upper leg. and the knce is accelerating $a_{k}$ relative to the ankle, the acceleration of the hip relative to ankle would be the vector addition of $a_{h}+a_{k}$.

The inertial resistance torque is due to rotation of a body. If a mass is not rotating, the point of mass concentration is at its center of gravity. If it is rotating, then this point changes and is called the center of gyration. As a result, the inertial force acts at the center of gyration rather than the center of gravity. Therefore, the inertial resistance moment. $M_{l}$, gives the additional moment about the axis of rotation due to a shift in the point of mass concentration. Assuming the moment of inertia, $J$, (which is a function of the way the mass is distributed in the link) stays constant

$$
M_{l}=I \ddot{x}
$$

The moment of inertia, $l$, can be expressed as follows

$$
I_{i}=\frac{W_{i}}{\mathrm{~g}}\left[F_{\mathrm{rg}}^{2}-F_{i \mathrm{~g}_{1}}^{2}\right] L L_{i}
$$

where $L L_{i}$ is the length of link $i, F_{\mathrm{rg}}$ is the fraction of link 


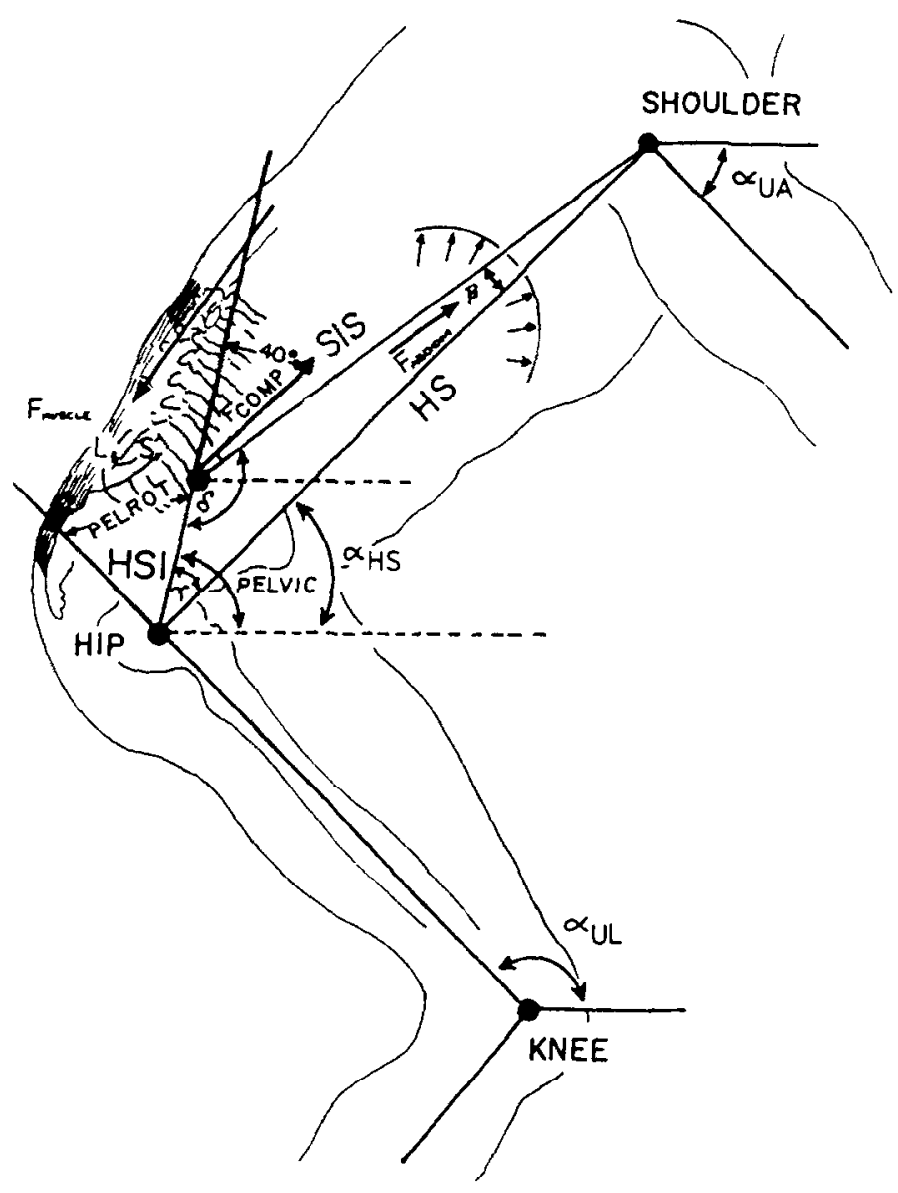

Fig. 9. $L_{s} / S_{1}$ joint derivation.

length to the radius of gyration, obtained from Plagenhoef (1963). and $F_{\text {a }}$, is the fraction of link length to the link center of gravity, obtained from Dempster (1955). Thus the forces and moments at a joint due to motion of a link can be determined by using the link weight, the linear acceleration of the eenter of gravity, the angular acceleration of the link, and the moment of inertia of the link, and the link length.

In addition to the forces on the body due to motion, there exist forces due to the effect of gravity on the load and mass of body links calculated in the fourth step. Figure I shows a freebody diagram of the body demonstrating all the forces and moments that exist on the body in an instantaneous position during a lift. As an example. the reactive force at the wrist is

$$
R_{w}=\left(F_{h u_{a}} \overline{i+}+\left(F_{h u_{v}}+W_{h d}\right) \bar{j}\right.
$$

Similar calculations can be performed for the other articulations.

Since the main interest of the research project is to examine factors in $\mathrm{MMH}$ activities that may have back compressive forces, special care was devoted to the calculation of reactive forces and torques at the $L_{s} / S_{1}$ joint. Furthermore, several assumptions had to be developed for these calculations.

In finding the forces on the spine. equilibrium equations are used in a similar way. The rg of the trunk and head is located on the trunk approximately on a line between the shoulder and hip as shown in Fig. 9 (Dempster, 1955). The assumption is made that this holds true throughout a lifting motion. As a result, the motion of the center of gravity of the trunk and head is not affected by the movement of the spine and the magnitude of the inertial fore is dependent only on mass and acceleration. However, the trunk mass including head and neck above the $L_{5} / S_{1}$ disc cannot be measured easily. It has been estimated to be $34.3 \%$ of the total body weight or about $62 \%$ of the trunk weight (Morris et al., 1961).

The reactive forces of the discs are used to calculate the compressive (normal) and shearing (tangential) loads as in Fig. 9. In addition to normal forces on the $L_{5} / S$ disc as a result of external forces, the erector spinae muscles exert a normal force to counteract the moment around the discs. This force is also coupled to the spine, and as such is added to the normal force on the disc. The distance, $d$, that the muscles act behind the nucleus of the disc has been established to be about $5 \mathrm{~cm}$ (Bartelink, 1957; Perey, 1957).

It has been established that the abdominal muscles are active in lifting actions, and as a result the abdomen exerts pressure on the pelvis and diaphragm which helps the spine carry the load (Bartelink, 1957; Davis and Troup, 1964: Morris et al., 1961). Using the data of Morris et al. (1961). some estimates could be made on the magnitude of the abdominal pressure during lifting. These have been regressed on the amount of moment on the hip during lifting (Chaffin. 
1969). The resulting regression equation is

$$
\begin{aligned}
P_{u b d o m}= & 0.01333\left[0.6516-0.005417\left(x_{L L}-x_{H S}\right)\right] \\
& \times\left(10.19 . M_{\text {hip }}\right)^{1.8} .
\end{aligned}
$$

An upper limit on $P_{\text {atdom }}$ is established at $20000 \mathrm{~N} \mathrm{~m}^{-2}$ (150 mmHg) from Bartelink (1957).

To find the force and moment exerted by the abdomen, the pressure $P_{\text {abdom }}$ is multiplied by the surface area of the diaphragm which Morris et al. (1961) have estimated to be $0.0465 \mathrm{~m}^{2}$. The distance this force acts anterior to the spine varies with the amount of trunk rotation. and can be estimated from Morris et al. (1961), to be

$$
m u_{a b d u m}=0.067+0.082 \sin \left(x_{L L}-\alpha_{H S}\right) .
$$

Thus

$$
F_{a b d o m}=P_{a b d o m} \times 0.0465
$$

and

$$
M_{a b d o m}=F_{a b d o m} \times m a_{a b s o m} .
$$

$F_{\text {comp }}$, then. can be calculated from the equilibrium equations around the center of rotation of the $L_{5} / S_{1}$ joint, which is centered geometrically in the disc between the $L_{9}$ and $S_{1}$ vertebrae. (Note. that the force acting on the $L_{5} / S_{1}$ disc is adjusted by $40^{\circ}$ to account for the orientation of the disc).

$\sum M=\sum r \times F=0.05 \times F_{\text {muscle }}$

$$
+M_{\text {abdom }}-M L_{5} / S_{1}=0
$$

$\sum F=F_{\text {comp }}+F_{\text {abdom }}-F_{\text {muscle }}$

$$
-F L_{3} S_{1} \times \sin \left(\text { pelvic }-40^{\circ}\right)=0 \text {. }
$$

(The force acting on the $L_{s} / S_{1}$ disc is adjusted by $40^{3}$ to account for the orientation of the disc.) 